\title{
ANTIOXIDANTES NA MANUTENÇÃO DO EQUILÍBRIO REDOX CUTÂNEO: USO E AVALIAÇÃo DE SUA EFICÁCIA
}

\author{
Thais Guaratini, Marisa H. G. Medeiros e Pio Colepicolo* \\ Departamento de Bioquímica, Instituto de Química, Universidade de São Paulo, CP 26077, 05513-970 São Paulo - SP, Brasil
}

Recebido em 12/12/05; aceito em 13/2/06; pleblicado na web em 30/8/06

\begin{abstract}
ANTIOXIDANTS IN THE SKIN: APPLICATIONS AND EVALUATION OF THEIR EFFICACY. The skin is a very complex organ, continuously exposed to physical, chemical and microbiological agents. Enzymes as well as low-molecular weight antioxidants are present in the cutaneous tissue to counterbalance the deleterious effect caused by an oxidative stress and thus maintain homeostasis. Antioxidants such as vitamins $\mathrm{C}$ and $\mathrm{E}$, carotenoids, and extracts with these properties have been extensively used for treatment of pathologies and skin aging prevention. We review here different mechanisms that can interfere in the redox equilibrium of the skin, as well as the chemical reactions involved in these processes. Moreover, we discuss the importance of endogenous or exogenous antioxidants that can be acquired from the diet or from oral or topical administration, and methodologies that have been developed to evaluate their efficacy
\end{abstract}

Keywords: skin, antioxidants; efficacy.

\section{INTRODUÇÃO}

A pele é um órgão complexo, formado por diferentes estruturas e com diversos tipos celulares (Figura 1), que atua como uma barreira protetora dos órgãos internos ao ambiente, enquanto mantém o balanço entre proliferação e descamação celular ${ }^{1}$. Mais que qualquer outro tecido, é exposta a inúmeros agentes químicos, físicos e microbiológicos, muitos dos quais induzem à formação de espécies reativas de oxigênio (ERO) e de nitrogênio (ERN). Estas espécies são fundamentais em diversos processos fisiopatológicos e bioquímicos, mantendo a sobrevivência e a homeostase celular, sendo que há um equilíbrio refinado entre sua formação e remoção. Porém, quando há alterações acentuadas neste equilíbrio, um estado pró-oxidante é gerado, levando assim ao chamado estresse oxidativo $^{2}$.

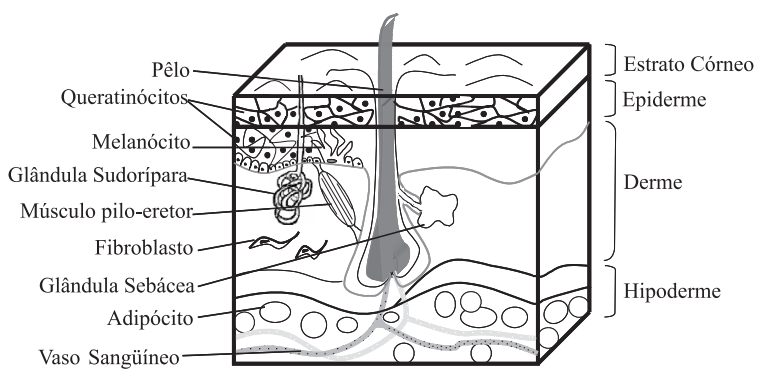

Figura 1. Estrutura esquemática da pele

Neste contexto, fica claro que a pele, por sua importância, é um órgão cujas células possuem mecanismos enzimáticos de resposta rápida, bem como moléculas antioxidantes de baixo peso molecular para contrabalançar o efeito deletério causado por um estresse oxidativo ${ }^{2}$, como apresentado na Figura 2.

Dentre as ERO formadas na pele, podemos destacar os radicais hidroxila $\left(\mathrm{HO}^{\circ}\right)$ e superóxido $\left(\mathrm{O}_{2}^{-}\right)$, os radicais peroxila e alcoxila

*e-mail: piocolep@iq.usp.br

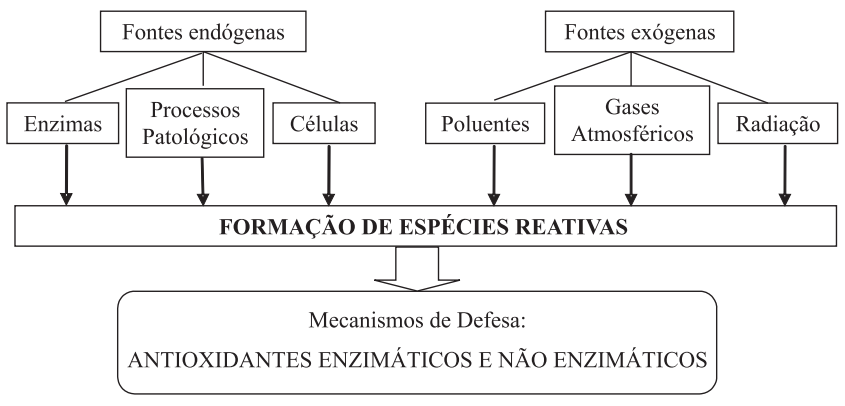

Figura 2. Fontes de espécies reativas na pele e mecanismos de defesa

$\left(\mathrm{RO}_{2} \cdot\right.$ e RO$\left.\cdot\right)$, o oxigênio singlete $\left({ }^{1} \mathrm{O}_{2}\right)^{3-5}$ e os peróxidos de hidrogênio $\left(\mathrm{H}_{2} \mathrm{O}_{2}\right)$ e orgânicos $(\mathrm{ROOH})$. Além das ERO, também estão envolvidas em processos redox outras espécies intermediárias, as espécies reativas de nitrogênio (ERN), tais como $\mathrm{NO}$ e espécies reativas de enxofre, com importância biológica significativa ${ }^{6}$.

As reações de redução completa do $\mathrm{O}_{2}$ requerem a transferência de quatro elétrons, que ocorrem diretamente nos complexos enzimáticos encontrados na membrana mitocondrial, resultando na formação de $\mathrm{H}_{2} \mathrm{O}$. Porém, é sabido que pode ocorrer também uma transferência univalente de elétrons, envolvendo a formação de intermediários radicalares ou não, pela redução incompleta do $\mathrm{O}_{2}$ na mitocôndria durante a cadeia de transporte de elétrons ${ }^{6}$, como mostra o Esquema 1. Além disso, hipóxia, isquemia-reperfusão, pro-

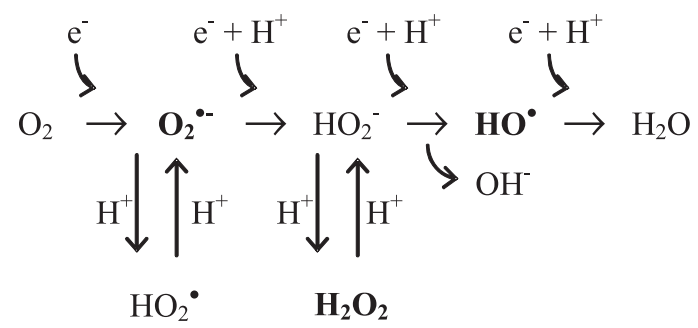

Esquema 1. Redução do $\mathrm{O}_{2}$ pela transferência univalente de elétrons e formação de intermediários reativos 
cessos do ciclo redox como a oxidação de quinonas e metabolismo de xenobióticos, entre outros, também podem gerar essas ERO? ${ }^{7}$.

$\mathrm{O}_{2}{ }^{*}$ é uma espécie radicalar, originado da adição de um elétron no orbital $\pi$ anti-ligante do $\mathrm{O}_{2}$, que não atravessa membranas, exceto por alguns canais específicos, ou quando apresentado em sua forma protonada ${ }^{6}$ (Esquema 2). Este radical pode ser gerado espontaneamente em condições controladas nas células por diferentes processos, tais como oxidação de hemoglobinas nos eritrócitos, atividade oxidativa do citocromo $\mathrm{P}_{450}$, reações de catálise realizada pela xantina oxidase, bem como o próprio processo de respiração mitocondrial, entre outros ${ }^{8}$.

$$
\mathrm{HO}_{2} \stackrel{\mathrm{H}^{+}}{=} \mathrm{H}^{+}+\mathrm{O}_{2}^{.-} \quad \mathrm{pKa}=4,8
$$

Esquema 2. Equilíbrio químico do radical $\mathrm{O}_{2}$.

Por suas características químicas, o $\mathrm{O}_{2}{ }^{--}$pode induzir desprotonações ou transferência de elétrons, além de atuar como um potente nucleófilo ${ }^{6}$. Porém, sua velocidade de reação com DNA, lipídeos e aminoácidos em solução aquosa $(\mathrm{pH}=7,4)$ é muito baixa, sendo que os danos biológicos pelo qual é responsável freqüentemente envolvem sua reação com outros radicais, com grupamentos ferro-enxofre de proteínas, bem como sua indução indireta na formação do radical $\mathrm{HO}^{*}$ pela redução de metais de transição, como ferro ou cobre ${ }^{6}$ (Esquema 3).

$$
\begin{array}{ll}
\mathrm{Fe}^{3+} / \mathrm{Cu}^{2+}+\mathrm{O}_{2}^{-} \rightarrow \mathrm{Fe}^{2+} / \mathrm{Cu}^{+}+\mathrm{O}_{2} & \begin{array}{l}
\mathrm{O}_{2}^{*-} \text { pode reciclar os metais de } \\
\text { transição para a reação de Fenton }
\end{array} \\
\mathrm{Fe}^{2+} / \mathrm{Cu}^{+}+\mathrm{H}_{2} \mathrm{O}_{2} \rightarrow \mathrm{Fe}^{3+} / \mathrm{Cu}^{2+}+\mathrm{HO}^{-}+\mathrm{HO}^{-} & \text {Reação de Fenton } \\
\mathrm{O}_{2}{ }^{-+}+\mathrm{H}_{2} \mathrm{O}_{2} \stackrel{\mathrm{Fe}^{2+} / \mathrm{Cu}^{+}}{\rightarrow} \mathrm{O}_{2}+\mathrm{HO}^{-}+\mathrm{HO}^{-} & \text {Reação de Haber-Weiss }
\end{array}
$$

Esquema 3. Reações de $\mathrm{O}_{2}{ }^{-}$e $\mathrm{H}_{2} \mathrm{O}_{2}$ contribuindo para a formação de $\mathrm{HO}^{*}$

A dismutação espontânea ou catalisada enzimaticamente de $\mathrm{O}_{2}^{-{ }^{-}}$, bem como o metabolismo mitocondrial e a atividade de algumas enzimas, como xantina, urato e aminoácidos oxidases, podem originar $\mathrm{H}_{2} \mathrm{O}_{2}$. Esta espécie é um fraco agente oxidante e redutor, que não reage diretamente com DNA, lipídeos e a maioria das proteínas ${ }^{6,9}$, porém, é difusível entre membranas e pode originar o radical $\mathrm{HO}^{\circ}$, pela reação de Fenton (Esquema 3). Além disso, seus alvos diretos incluem enzimas como a gliceraldeído-3-fosfato-desidrogenase, cetoácidos como o piruvato e o oxoglutarato e grupos heme ${ }^{6}$.

O radical HO;, por sua vez, é bastante reativo, podendo abstrair hidrogênios de outras moléculas, reagir com compostos por adição, ou ainda transferir seus elétrons. Os danos genéticos provocados pela radiação UV envolvem não somente a absorção de energia direta pelo DNA, como também a formação de radical $\mathrm{HO}^{\circ}$, que reage com esta biomolécula, podendo gerar modificações mutagênicas, estando assim envolvido nos mecanismos de indução do câncer ${ }^{6,10,11}$.

$\mathrm{O}^{1} \mathrm{O}_{2}$ é outra ERO envolvida em processos fisiológicos e patológicos. Esta espécie eletronicamente excitada pode ser produzida por diversas reações químicas e fotoquímicas, e devido à sua eletrofilicidade, pode reagir com compostos insaturados, sulfetos e aminas, sendo que ácidos graxos insaturados, DNA e proteínas representam importantes alvos biológicos ${ }^{12-15}$.

O equilíbrio entre a formação e a remoção das espécies citadas pode sofrer ação de agentes exógenos ou endógenos, induzindo um estado de estresse oxidativo. Este, por sua vez, pode ser restabelecido pelos sistemas antioxidantes presentes no tecido cutâneo.

\section{BALANÇO REDOX NA PELE}

O sistema antioxidante cutâneo é formado por substâncias enzimáticas e não-enzimáticas. Dentre os antioxidantes enzimáticos, destacam-se a glutationa peroxidase (GPx), a catalase (CAT) e a superóxido dismutase (SOD) ${ }^{16}$.

A SOD, que contribui para um dos mecanismos antioxidantes mais eficientes, converte $\mathrm{O}_{2}{ }_{2}^{--}$em peróxido de hidrogênio $\left(\mathrm{H}_{2} \mathrm{O}_{2}\right)$, prevenindo os danos que poderiam ser causados por este radical (Esquema 4). Diversos tipos de SOD são descritos na literatura, podendo estar localizadas no citosol, em organelas celulares específicas ou extracelularmente, e cujos sítios ativos podem conter diferentes íons (como cobre, zinco, manganês ou ferro) ${ }^{17}$.

$$
2 \mathrm{O}_{2}^{--}+2 \mathrm{H}+\stackrel{\text { SOD }}{\rightarrow} \mathrm{H}_{2} \mathrm{O}_{2}+\mathrm{O}_{2}
$$

Esquema 4. Dismutação do $\mathrm{O}_{2}^{\circ-}$ pela enzima SOD

A CAT e a GPx são as principais responsáveis pela remoção imediata de $\mathrm{H}_{2} \mathrm{O}_{2}$. A CAT, encontrada em peroxissomos, catalisa a decomposição específica de $\mathrm{H}_{2} \mathrm{O}_{2}$ gerando moléculas de água e oxigênio $^{17}$ (Esquema 5). A GPx, por sua vez, é fundamental no metabolismo de $\mathrm{H}_{2} \mathrm{O}_{2}$ e de outros peróxidos, pois catalisa reações de doação de elétrons, no qual se utiliza da glutationa reduzida (GSH) como agente redutor, formando a glutationa oxidada (GSSG) ${ }^{18}$ (Esquema 5).

$$
\begin{aligned}
& 2 \mathrm{H}_{2} \mathrm{O}_{2} \stackrel{\text { CAT }}{\rightarrow} 2 \mathrm{H}_{2} \mathrm{O}_{2}+\mathrm{O}_{2} \\
& \mathrm{H}_{2} \mathrm{O}_{2}+2 \mathrm{GSH} \stackrel{\mathrm{GPx}}{\rightarrow} 2 \mathrm{H}_{2} \mathrm{O}+\mathrm{GSSG}
\end{aligned}
$$

$$
\mathrm{LOOH}+2 \mathrm{GSH} \stackrel{\mathrm{GPx}}{\rightarrow} 2 \mathrm{H}_{2} \mathrm{O}+\mathrm{GSSG}+\mathrm{LOH}
$$

Esquema 5. Degradação enzimática de $\mathrm{H}_{2} \mathrm{O}_{2}$ e de peróxidos orgânicos (GSH: glutationa reduzida; GSSG: glutationa oxidada; LOOH: hidroperóxido lipídico e LOH: o produto de redução correspondente)

Os antioxidantes não-enzimáticos, ou de baixo peso molecular também contribuem com a manutenção do balanço redox celular. Nesta classe inclui-se um vasto número de compostos, sintetizados in vivo ou obtidos exogenamente, que previnem danos oxidativos por interações diretas ou indiretas com as ERO/ERN ${ }^{19,20}$.

Dentre as substâncias endógenas podemos destacar alguns hormônios, como estradiol e estrógeno que apresentam atividade antioxidante semelhante à vitamina E, devido, provavelmente, à sua porção fenólica, comum a ambas as moléculas ${ }^{21}$ e a melatonina, reguladora do relógio biológico nos mamíferos, que também apresenta atividade antioxidante, provavelmente devido à desidrogenação do grupamento - $\mathrm{NH}$, além de induzir a síntese de antioxidantes enzimáticos in vivo, como a glutationa peroxidase ${ }^{22,23}$. Destacam-se também o ácido lipóico, um cofator essencial em vários complexos enzimáticos que apresenta atividade antioxidante e que pode atuar como regenerador de formas oxidadas de glutationa, ascorbato e $\alpha$-tocoferol ${ }^{24}$; bem como a melanina, um pigmento formado pela oxidação e polimerização da tirosina, com papel protetor contra a radiação UV, que também possui papel antioxidante, protegendo a pele principalmente contra $\mathrm{O}_{2}{ }^{--}$e $\mathrm{RO}_{2}^{25}$ (Figura 3). 

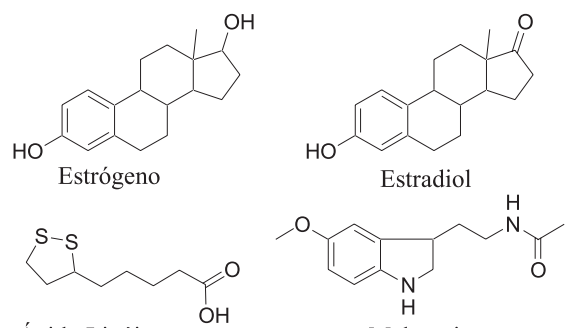

Ácido Lipóico
$\mathrm{OH}$

Melatonina
Figura 3. Estrutura química de alguns dos antioxidantes cutâneos sintetizados in vivo

Além de todo o sistema antioxidante endógeno exemplificado, a pele conta ainda com um eficiente mecanismo de reparo, caso os danos já tenham ocorrido, envolvendo enzimas e substâncias de baixo peso molecular ${ }^{26}$. Por ex., lesões oxidativas em DNA podem ser identificadas por enzimas específicas, removidas e reparadas ${ }^{27}$, ou ainda, substâncias que sofreram ataque de radicais podem ser restauradas pela doação de hidrogênio de outras moléculas ${ }^{26}$.

\section{ALTERAÇÕES REDOX MEDIADAS PELA EXPOSIÇÃO SOLAR}

Diversos fatores podem iniciar ou contribuir para alterações no equilíbrio redox na pele, sendo que muitos aceleram o envelhecimento intrínseco cutâneo, ou seja, o envelhecimento cronológico inerente a todos os órgãos. A exposição solar é um dos fatores mais importantes, por seus efeitos cumulativos, podendo ter como conseqüência o câncer de pele ${ }^{26}$.

A radiação UV pode ser dividida em três faixas, considerandose suas características de propagação e efeitos biológicos: UVC (100-290 nm) que praticamente não chega à superfície da Terra por ser filtrada pela camada de ozônio; UVB (290-320 nm) que devido à sua alta energia é responsável pelos danos imediatos da radiação solar; e UVA (320-400 nm), que induz alterações celulares, sendo os fibroblastos e os melanócitos particularmente susceptíveis ${ }^{28,29}$. Assim, no estudo das respostas cutâneas à radiação UV, deve ser avaliado o comprimento de onda em questão, a dose, e características intrínsecas, como por ex., susceptibilidade genética e pigmentação da pele ${ }^{30}$.

As radiações UVA e UVB são responsáveis pela diminuição de sistemas antioxidantes cutâneos, bem como pelo aumento de sistemas oxidantes, por diversos mecanismos, alterando assim o balanço redox celular e, conseqüentemente, a homeostasia cutânea ${ }^{31-35}$. A primeira resposta observada na pele após exposição ao sol é a inflamação, caracterizada por eritema, edema e calor, e pela elevação dos níveis de prostaglandinas e leucotrienos. Células especializadas, como neutrófilos, são recrutados e estimulados, seguido da ativação do sistema NAD $(\mathrm{P}) \mathrm{H}$ oxidase, gerando uma série de espécies reativas ${ }^{36-}$ ${ }^{38}$. Como resultado, há uma alteração no sistema imunológico, diminuindo sua capacidade de eliminar células alteradas, devido às mudanças na produção de citocinas pelos queratinócitos e outras células cutâneas, alterações na expressão de moléculas de adesão e perda de funções celulares ${ }^{36,39}$.

Além da inflamação, outros mecanismos de resposta podem originar espécies reativas na pele. Heck e colaboradores ${ }^{40}$ identificaram uma catalase presente em queratinócitos capaz de formar ERO em resposta à radiação UV, em especial, UVB. Kuhn e colaboradores ${ }^{41}$ também demonstraram uma expressão aumentada de óxido nítrico sintase (NOS) na pele quando exposta à radiação UVB, resultando em formação excessiva de óxido nítrico ( $(\mathrm{NO})$, que tem o papel fisiológico de estimular os melanócitos a produzirem mais melanina ${ }^{6}$. Além disso, o ${ }^{*} \mathrm{NO}$ pode reagir com $\mathrm{O}_{2}^{--}$formando peroxinitrito $\left(\mathrm{ONOO}^{-}\right)$, uma espécie altamente reativa ${ }^{42,43}$. As espécies reativas podem ser formadas ainda por reações da luz com fotossensibilizadores endógenos cutâneos na presença de $\mathrm{O}_{2}$, como por ex., o ácido urocânico ${ }^{44,45}$.

A reação das espécies reativas então formadas, com componentes celulares, pode resultar em lipoperoxidação, oxidação de proteínas $^{46,47}$ e de DNA. A indução da peroxidação de ácidos graxos pode induzir à conversão de vários deles em hidroperóxidos, por reações em cadeia (Esquema 6). Um dos produtos formados destas reações é o malonaldeído (MDA), composto reativo que pode atacar grupamentos amino de proteínas e fosfolipídios, modificando moléculas biológicas e ativando uma resposta auto-imune ${ }^{48}$. Os aldeídos formados pela lipoperoxidação podem ainda interagir com o DNA, formando adutos que têm sido associados com câncer ${ }^{49,50}$.

$$
\begin{array}{ll}
\text { Início } & \mathrm{LH}+\mathrm{OH}^{\bullet} \rightarrow \mathrm{L}^{\bullet}+\mathrm{H}_{2} \mathrm{O} \\
\text { Propagação } & \mathrm{L}^{\bullet}+\mathrm{O}_{2} \rightarrow \mathrm{LOO}^{\bullet} \\
\text { Propagação } & \mathrm{LH}+\mathrm{LOO}^{\bullet} \rightarrow \mathrm{L}^{\bullet}+\mathrm{LOOH}
\end{array}
$$

Esquema 6. Mecanismo de início e propagação da lipoperoxidação (LH: ácido graxo insaturado; $L^{*}$ : radical lipídico; $L O O^{\circ}$ : radical peroxila e $L O O H$ : hidroperóxido lipídico)

Os danos diretos, ou mediados pelas espécies reativas que ocorrem ao DNA, disparam respostas celulares como ativação de sistemas de reparo e enzimas controladoras do ciclo celular ${ }^{51}$. Esses intermediários e seus metabólitos podem, então, reagir com eletrófilos celulares, alguns dos quais iniciam um processo de sinalização celular, modificando a expressão gênica ${ }^{52}$.

Há evidências de que em peles com exposição crônica ao sol há maior incidência de mutações no gene supressor de tumor p53, pelo estresse oxidativo ocasionado ${ }^{53,54}$. Mutações neste gene, responsável pela manutenção da integridade genômica por bloquear a replicação do DNA em resposta a danos, ou induzir seu reparo ${ }^{55}$, reduzem sua capacidade em disparar a morte programada de células (apoptose), resultando em maior proliferação celular ${ }^{56}$. Sugerese ainda que, independentemente dos danos causados ao DNA, o desequilíbrio redox provocado pela radiação UV pode interferir na apoptose $^{57-59}$

Assim, os efeitos deletérios da radiação UV na pele são cada vez mais evidentes. Além disso, os mecanismos descritos, como transduções de sinais, mutações em genes específicos, alterações na proliferação celular e no mecanismo de apoptose, podem exemplificar os passos do complexo processo carcinogênico, que é a principal preocupação quanto à exposição à radiação UV e seus metabólitos.

Tendo em vista a prevenção ou terapias para o câncer de pele, que é um dos tipos de câncer de maior ocorrência, são muito discutidos mecanismos para o restabelecimento do equilíbrio redox, sendo que o uso de antioxidantes nesses casos é uma alternativa, que tem sido amplamente utilizada, apesar das divergências encontradas ${ }^{60}$.

\section{USO DE SUBSTÂNCIAS ANTIOXIDANTES E SUA RELAÇÃO RISCO-BENEFÍCIO}

A aplicação tópica ou oral de antioxidantes enzimáticos ou nãoenzimáticos representa uma estratégia interessante de proteção cutânea contra o estresse oxidativo ocasionado por diferentes agentes. Além da reposição utilizando moléculas endógenas, buscam- 
se novas substâncias com ação antioxidante, bem como substâncias que irão aumentar direta ou indiretamente os níveis endógenos dos antioxidantes.

Alguns antioxidantes fundamentais são normalmente adquiridos pela dieta, como por ex., as vitaminas $\mathrm{C}$ e E, os carotenóides e substâncias fenólicas derivadas de plantas (Figura 4). A vitamina E, ou $\alpha$-tocoferol, além de estabilizar as bicamadas lipídicas no estrato córneo, é um dos mais importantes inibidores da peroxidação lipídica em animais, por capturar $\mathrm{RO}_{2}{ }^{\cdot 61}$. Glândulas sebáceas são as responsáveis pela sua secreção na superfície cutânea, bem como de outros tocoferóis e tocotrienóis ${ }^{11}$, sendo que a região facial apresenta maior concentração desta substância, coerente à maior exposição a agentes externos nesta área ${ }^{62}$.

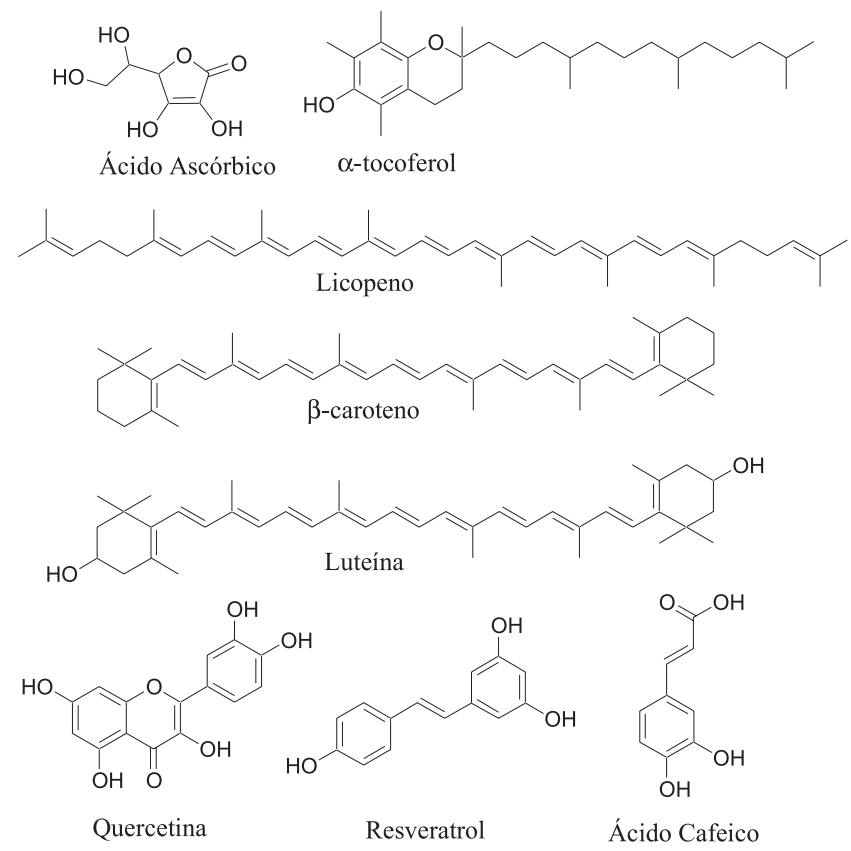

Figura 4. Exemplos de substâncias antioxidantes obtidas pela dieta: vitaminas $C$ (ácido ascórbico) e E ( $\alpha$-tocoferol), carotenóides (licopeno, $\beta$-caroteno $e$ luteína) e compostos fenólicos (quercetina, ácido cafeico e resveratrol)

Inúmeros são os trabalhos relatando que o $\alpha$-tocoferol pode atenuar o estresse oxidativo, principalmente por proteger membranas contra a lipoperoxidação ${ }^{61,63-66}$. Assim, por ter sua eficácia comprovada, esta vitamina é muito utilizada na prevenção de doenças ou fotoenvelhecimento da pele, sendo veiculada tanto em suplementos orais, como em produtos de uso tópico. Em suplementos orais, sua biodisponibilidade cutânea após a ingestão deve ser considerada. Além da biotransformação que pode ocorrer durante a digestão, outros fatores, como a composição da dieta, podem interferir na biodisponibilidade dos suplementos administrados por via oral. No caso desta vitamina, por ser uma molécula lipossolúvel, a ingestão concomitante de gordura é fundamental para que seja absorvida e, assim, possa exercer sua atividade.

Estudos mostram que voluntários suplementados com cápsulas de $\alpha$-tocoferol deuterado (150 mg por dia), juntamente com ingestão de lipídios, têm a vitamina detectada na pele após 7 dias. Os vários passos bioquímicos e fisiológicos necessários para a liberação cutânea do $\alpha$-tocoferol não são ainda conhecidos ${ }^{66}$.

Vários produtos fotoprotetores ou para aplicação após exposição ao sol contêm o $\alpha$-tocoferol, ou seu éster, como princípio ativo coadjuvante, por inibir não somente a lipoperoxidação como também a formação de dímeros de timina e a imunossupressão ${ }^{67,68}$. Em contrapartida, recentemente foi verificado que esta vitamina (e o seu éster), em concentrações abaixo ainda da encontrada em tais formulações, pode inibir a glutationa-S-transferase (GST), sendo que a ausência desta enzima, que é responsável pela detoxificação de compostos citotóxicos, como os aldeídos resultantes da lipoperoxidação, aumenta o risco de câncer de pele ${ }^{69}$. Estes dados confirmam os dados obtidos por Mitchel e McCann, que detectaram em 1993, uma atividade promotora de tumor pela vitamina $\mathrm{E}^{70}$.

Outros metabólitos da vitamina E, como quinonas ou produtos da oxidação de sua cadeia ${ }^{69,71}$, também foram identificados e estudados, sendo que o risco do uso desta substância por via oral ou tópica deve ser considerado, e a relação custo-benefício avaliada antes de seu uso indiscriminado.

A administração de antioxidantes em combinação parece ser uma estratégia de tratamento mais efetivo. Este sinergismo pode ser muito bem exemplificado pelo uso concomitante das vitaminas E e C. A vitamina C (ácido ascórbico), cofator de diversas enzimas e essencial na síntese de colágeno, regenera o radical tocoferila formado na reação do $\alpha$-tocoferol com radicais e atua como um antioxidante in vivo, fazendo parte da linha de defesa hidrossolúvel ${ }^{72}$. Em estudos com voluntários foi verificado que o uso da vitamina $\mathrm{E}$ apresenta maiores efeitos benéficos quando administrada em conjunto com a vitamina $\mathrm{C}$, pelo fato de ambas atuarem sinergisticamente, sendo que a vitamina $\mathrm{C}$ pode reciclar o radical tocoferila (Esquema 7) e a combinação dessas vitaminas podem aumentar efetivamente a atividade antioxidante total da pele p3-75. $^{7}$.

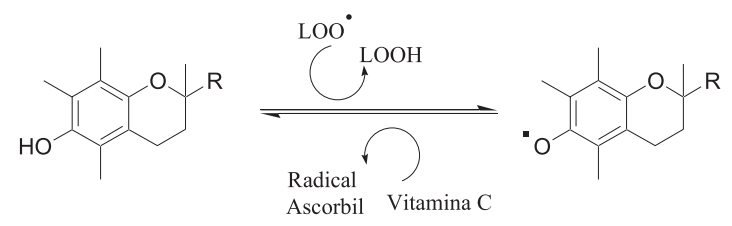

Esquema 7. Reação do $\alpha$-tocoferol (sendo $R$, a sua cadeia lateral) com radicais peroxila (LOO*), formando hidroperóxidos lipídicos (LOOH) e sua regeneração pela vitamina $C$, originando o radical ascorbila

O ácido ascórbico quando administrado isoladamente também atua contra os danos induzidos pela radiação UV, capturando as espécies reativas geradas ${ }^{34,76,77}$. Esta vitamina está presente na forma de ânion ascorbato em pH fisiológico, sendo que este apresenta menor penetração em comparação à sua forma esterificada (palmitato de ascorbila), que é mais lipossolúvel ${ }^{78}$. Estudos mostram que a aplicação de uma formulação contendo ascorbato pode aumentar até em 25 vezes a sua concentração na pele ${ }^{79}$, contudo a suplementação oral não mostra efeitos benéficos significativos na pele ${ }^{80}$.

Por outro lado, a vitamina $\mathrm{C}$, assim como o $\alpha$-tocoferol, pode atuar como pró-oxidante, pois por ser um forte agente redutor, pode reagir com metais de transição, reciclando-os para a reação de Fenton (Esquema 3) e facilitando a formação dessas espécies ${ }^{81}$. Porém, apesar dos efeitos adversos constatados, autores afirmam que tanto sua forma ácida quanto as diversas formas esterificadas utilizadas atualmente, apresentam-se seguras para utilização tópica ou oral ${ }^{82}$.

Atuando sinergisticamente com as vitaminas $\mathrm{C}$ e $\mathrm{E}^{83}$, estão os carotenos e as xantofilas (oxocarotenos), presentes em folhas, frutos e legumes frescos ${ }^{84,85}$ e que se concentram nas membranas celulares protegendo-as da lipoperoxidação, por interagirem com $\mathrm{RO}_{2}{ }^{\cdot 86-88}$. Os carotenóides são conhecidos ainda pela sua eficiência em desativar ${ }^{1} \mathrm{O}_{2}$ e espécies tripletes de moléculas fotossensibilizadas, além de sua habilidade em interagir com outras espécies reativas, formando adutos ou carotenóides radicalares, inibindo processos oxidativos ${ }^{89}$.

A eficácia dos carotenóides está relacionada ao número de li- 
gações duplas conjugadas na molécula, sendo o licopeno o mais eficiente $^{90,91}$. Sua atividade antioxidante é dependente da tensão parcial de $\mathrm{O}_{2}$ presente no meio. As baixas pressões de $\mathrm{O}_{2}$, como a encontrada na maioria dos tecidos, inibem a oxidação, porém, podem ser pró-oxidantes em maiores concentrações deste gás ${ }^{92-94}$. O efeito pró-oxidante observado pode estar relacionado aos efeitos adversos detectados em suplementações com altas doses de $\beta$ caroteno $^{93}$, ou ainda, aos produtos de degradação do $\beta$-caroteno, não sendo elucidado o mecanismo completo desta toxicidade ${ }^{95}$. Contudo, apesar dos resultados parecerem contraditórios na literatura, o uso como suplemento oral é muito difundido.

Ao lado do uso oral, a aplicação tópica de carotenóides também é uma maneira eficaz de restabelecer o balanço redox cutâneo. Alguns trabalhos mostram sua atividade frente ao estresse oxidativo causado pela radiação UV, sendo que sua habilidade protetora aumenta quando aplicados na pele juntamente com vitaminas ${ }^{96}$. Por serem moléculas pouco estáveis, a veiculação dos carotenóides requer um desenvolvimento farmacotécnico elaborado, a fim de assegurar sua integridade na formulação.

Também para restabelecimento do equilíbrio redox cutâneo, bem como para prevenção ou tratamento de patologias causadas por estresse oxidativo, são utilizadas muitas classes de substâncias antioxidantes provenientes de produtos naturais. Muitos extratos são veiculados em formulações para uso tópico, tendo sua eficácia comprovada. Além disso, inúmeras são as formas orais de suplementação, como a ingestão de chás, cápsulas, decotos, entre outros. Diversos estudos descritos na literatura abordam o uso de antioxidantes oriundos de produtos naturais. A eficácia de antioxidantes desta origem é extensamente investigada, principalmente frente aos efeitos da radiação UV, sendo utilizados alguns extratos como o de Calendula officinalis ${ }^{97}$, de Polypodium leucotomos ${ }^{98}$, de Pothomorphe umbellata ${ }^{99}$, entre muitos outros, a fim de prevenir ou amenizar os efeitos causados pela radiação UV.

O chá verde, nome popular dado para a infusão obtida a partir da planta Camellia sinensis, é rico em catequinas, que são poderosos antioxidantes. Dentre os efeitos observados, podem-se citar a inibição da lipoperoxidação e dos danos causados ao DNA pelas ERO, a inibição da imunossupressão e da inflamação cutânea induzida pela radiação UV, a indução de apoptose nas células tumorais e inibição do crescimento do tumor induzido pela radiação $U^{100}$. Além de ser consumido por quase um terço da população mundial, encontra-se também em produtos para uso tópico, apresentando efeitos semelhantes ${ }^{101,102}$.

Outra planta muito utilizada é o Ginkgo biloba, que apresenta propriedades antiinflamatórias, imunomodulatórias e antioxidantes, e vem sendo clinicamente testada em desordens cutâneas ${ }^{103,104}$. Recentemente, foi demonstrada sua eficácia também no tratamento de pacientes com vitiligo, sendo que a ingestão de $40 \mathrm{mg}$ de seu extrato mostrou-se eficaz em controlar a doença, além de repigmentar áreas afetadas ${ }^{105}$.

Os antioxidantes naturais são promissores na prevenção do câncer de pele, pois muitos já mostraram seus efeitos anticarcinogênico e antimutagênico, além de baixa toxicidade ${ }^{106}$. Assim, o uso de substâncias antioxidantes, por via oral ou tópica, pode auxiliar os sistemas endógenos de proteção da epiderme, além de contribuir para prevenção de problemas a longo prazo $^{107}$.

\section{MÉTODOS ANALÍTICOS PARA A DETERMINAÇÃO DA EFICÁCIA DE ANTIOXIDANTES NA PELE}

A eficácia do uso de antioxidantes na pele pode ser medida por uma série de parâmetros físico-químicos e com diferentes objetivos, dependendo da finalidade do emprego. Por ser um assunto muito amplo, os resultados podem demonstrar um problema clínico imediato ou ser suporte para pesquisas científicas, as quais exigem um alto grau de detalhamento ${ }^{108}$.

Os estudos podem ser realizados in vitro ou in vivo, este último utilizando animais ou seres humanos. Os estudos in vitro apresentam como vantagem a possibilidade de estudos mais agressivos, contudo necessitam de extrapolação e muitas vezes não reproduzem o mesmo efeito in vivo ${ }^{109}$. Os estudos in vivo, por sua vez, têm como maior vantagem, a possibilidade de determinação da eficácia de uma substância diretamente em seu local de ação, além de originarem resultados mais próximos do real.

As técnicas utilizadas quando se realizam os testes in vivo podem ser invasivas ou não-invasivas ${ }^{110}$. As técnicas não-invasivas são normalmente associadas à análise das conseqüências do desequilíbrio redox, como mudanças nas características elásticas da pele, alterações na perda de água transepidérmica e conteúdo aquoso do estrato córneo, mudanças no pH e na coloração cutânea, além de alterações no fluxo sangüíneo ${ }^{111}$. Essas propriedades da pele são mensuradas utsando-se métodos de bioengenharia, que constitui uma técnica eficiente para verificação de modificações cutâneas frente ao uso de um antioxidante, a fim de reverter um quadro de estresse oxidativo ${ }^{108}$.

Por outro lado, os sistemas invasivos ou com tecidos humanos obtidos por biopsia (ex vivo) são voltados à compreensão do fenômeno e utilizam técnicas e equipamentos mais sofisticados, como cromatografia líquida ou gasosa acoplada à espectrometria de massas, quimiluminescência, geração de imagem por espectroscopia de ressonância de spin eletrônico ou por espectrometria de massas com ionização por MALDI.

$\mathrm{O}$ uso da técnica de quimiluminescência permite a determinação quantitativa dos efeitos na pele humana ${ }^{112}$. Em circunstâncias normais, os processos físiológicos levam à formação de radicais, resultando em emissão de fótons (na ordem de 20 a 40 contagens por s), contudo essas quantidades são muito baixas para serem detectadas por instrumentações correntemente empregadas ${ }^{113}$. No estresse oxidativo, os níveis de radicais são geralmente aumentados, permitindo assim sua detecção através da emissão de fotóns produzidos ${ }^{35,114,115}$ Para a realização destes testes, os voluntários selecionados são acomodados em uma sala escura, com o equipamento de excitação e leitura sobre uma superfície do corpo previamente selecionada. Ao redor desta área o tecido é protegido com fitas adesivas hospitalares para evitar estresse desnecessário em outras regiões. Inicialmente o aparelho faz a leitura dos níveis basais, para a obtenção do branco e, a seguir, uma energia no comprimento de onda selecionado (no caso do UVA, de $2 \mathrm{~J} / \mathrm{cm}^{2}$, liberado a 20 $\mathrm{mW} / \mathrm{cm}^{2}$ ) é aplicada e instantaneamente inicia-se a leitura pela célula fotomultiplicadora. Recentemente, foi demonstrado que o uso desta técnica permite o estudo da cinética de decaimento de fótons após o estímulo, construindo-se um modelo cinético que permite estudos comparativos entre pele total e estrato córneo ${ }^{113}$. Apesar de não ocorrerem lesões mecânicas nos voluntários como, por ex., micro-cirurgias, este procedimento é considerado invasivo, pois a radiação provoca danos, mesmo que pequenos e reversíveis.

Outro sistema com base no mesmo princípio de emissão de energia sobre a pele foi recentemente demonstrado ${ }^{116}$. Sugeriu-se pela primeira vez, o uso da técnica de espectroscopia de Raman para detecção de substâncias antioxidantes na pele humana, neste caso, $\beta$-caroteno e licopeno. O processo tem início com a emissão de um feixe de laser verde, o qual pode promover os estiramentos típicos dos sistemas poliênicos conjugados, sendo específico para carotenóides no experimento em questão. Por não levar a danos, nem resultar na formação de espécies reativas, este experimento foi considerado pelos autores como não-invasivo e permite sua aplicação em estudos de quantificação ${ }^{116}$. 
Dentre as técnicas invasivas, o sistema de microdiálise tem sido amplamente utilizado para os estudos onde se pretende avaliar a absorção de substâncias antioxidantes e possíveis metabólitos formados. Neste sistema, uma pequena sonda de membrana de policarbonato (aproximadamente $10 \mathrm{~mm}$ ) é introduzida cirurgicamente na região dérmica do corpo de um voluntário ${ }^{117}$. O material é aplicado topicamente na região acima da membrana por onde é perfundida uma solução (fisiológica ou de lactato, entre outras), a qual é recolhida em um amostrador para análise posterior. Normalmente as concentrações do material recolhido são extremamente baixas, o que muitas vezes limita o uso de sistemas de cromatografia líquida com detecção por ultravioleta. Neste modelo, o mais recomendado é o uso de detecção por espectrometria de massas, que por ser mais seletiva e sensível permite a quantificação das substâncias em estudo. Algumas moléculas, como o ácido ascórbico, são passíveis de análise por cromatografia gasosa ${ }^{118}$, contudo a maioria das substâncias antioxidantes é termolábil, o que impede sua análise por esta metodologia.

$\mathrm{Na}$ cromatografia líquida acoplada à espectrometria de massas, o sistema mais relatado na literatura para estudos de moléculas com estrutura poliênica apresentando propriedades antioxidantes é a ionização por APCI (ionização química à pressão atmosférica). Quando se trabalha com moléculas comerciais, o sistema de APCI é recomendado por possuir um menor número de parâmetros a serem controlados, permitindo estudos de liberação na pele de várias substâncias antioxidantes, incluindo a detecção de compostos marcados isotopicamente, como a vitamina $\mathrm{E}$ deuterada ${ }^{66}$. Contudo, quando estão sendo estudadas moléculas que não dispõem de quantidade de massa suficiente, em sistemas utilizando-se diretamente da bomba de infusão, essa técnica passa a ser limitante pois necessita de um fluxo alto $(1 \mathrm{~mL} / \mathrm{min})$ na fonte, sendo o sistema de ionização por "electrospray" (ESI) então recomendado, já que pode operar em fluxos muito baixos ( $10 \mu \mathrm{L} / \mathrm{min}$ na fonte), consumindo, portanto, muito menos amostra.

O maior problema no uso do sistema de ESI é a dificuldade de observarmos os íons quasi-moleculares esperados nesta técnica para alguns sistemas conjugados ${ }^{119}$. A análise de retinóides por APCI e ESI mostrou a presença de $[\mathrm{M}+\mathrm{H}]^{+}$ou eliminação neutra de água a partir da molécula protonada $\left[\mathrm{MH}-\mathrm{H}_{2} \mathrm{O}\right]^{+}$para vários deles no modo positivo, e retirada de $\mathrm{H}^{+}$para os seus derivados ácidos no modo negativo ${ }^{119,120}$. Por outro lado, em sistemas de solventes próticos, os carotenóides não apresentavam boas respostas nos experimentos de ESI, sendo que em experimentos com agentes oxidantes, o íon molecular ou bi-radicalar pode ser observado ${ }^{121}$. Em trabalho complementar, esses autores compararam o capilar da fonte a uma célula eletrolítica e sugeriram que a formação de íons moleculares seria relacionada aos potenciais redox das moléculas $^{122}$. Recentemente, foi definitivamente demonstrado que a ionização por ESI e MALDI de sistemas poliênicos similares ao $\beta$ caroteno apresentam relação direta entre formação de íon molecular e seu potencial redox, abrindo caminho para um novo uso analítico para a determinação das reações de metabolismo ${ }^{119}$. Em contraste, outros antioxidantes naturais, como flavonóides, derivados do ácido cafeico, resveratrol entre outros, não apresentam essa relação e são passíveis de análise em ambos os sistemas, APCI e ESI.

Alguns estudos visando compreender os locais onde ocorrem as reações deletérias podem ser realizados através de geração de imagens, porém, essas análises limitam os estudos a animais ou à pele retirada por biópsia. Trabalhos demonstram a utilidade da técnica de espectroscopia de ressonância de spin eletrônico (ESR) na observação de espécies radicalares em pele de ratos ${ }^{123}$. O desenvolvimento e a aplicação da técnica consistem na adição de substâncias que reagem efetivamente com os radicais, formando um novo radical mais estável e, portanto, com maior tempo de vida. A condição para a escolha dos reagentes ("spin trap") é que este seja diamagnético, para reação com o radical e posterior análise por ESR $^{123-125}$. Os nitróxidos estão sendo empregados para esses estudos, por reagirem rapidamente com ERO, sendo utilizados principalmente o 2,2,6,6,-tetrametilpiperidina-1-oxila (TEMPO), a 3carbamoil-2,2,5,5-tetrametilpirrolidina-1-oxila (PCM) e a 3carbonil-2,2,5,5-tetrametilpirrolidina-1-oxila (PCA) ${ }^{126}$. A pele após a reação com os nitróxidos é analisada em um sistema de gradiente de campo em duas dimensões e a diferença entre locais onde ocorreram reações (que indicam presença de radicais) permite a geração de uma imagem e, por conseqüência, a definição dos sítios reativos da pele. $\mathrm{O}$ mais recente estudo sistemático do emprego da técnica com as substâncias TEMPO, PCM e PCA demonstrou que essas moléculas podem ser empregadas em baixas concentrações (na ordem de $1 \mathrm{mM}$ ), sendo resistentes a processos de redução que podem interferir na análise ${ }^{126}$.

O mais novo sistema de análise por geração de imagem está sendo desenvolvido através do emprego da espectrometria de massas com ionização por MALDI, nomeada de MMI (imagem por espectrometria de massas). O princípio do método é auto-radiografia de cortes histológicos, onde ocorre uma varredura de pulsos de laser e acúmulo de espectros, sendo cada poço ou área de análise não superior a $100 \mu \mathrm{m}$. A reunião desses dados permite localizar a existência dos íons previamente selecionados de micromoléculas ou de proteínas com alta especificidade e geração de uma imagem. A imagem gerada, por sua vez, permite definir se os produtos de interesse estão no estrato córneo, derme, epiderme ou hipoderme ${ }^{127}$.

\section{CONCLUSÃO}

Os antioxidantes têm sido extensivamente utilizados tanto no tratamento de patologias, como na prevenção do envelhecimento cutâneo e das conseqüências causadas pela radiação UV em curto e longo prazo, como o câncer de pele.

Para conhecer sua eficácia é necessário analisar quais biomoléculas estão sendo protegidas, e se conseguem atingir o seu alvo, poupando o tecido ou não. Para isso, novas técnicas vêm sendo desenvolvidas visando a avaliação correta dos efeitos dos antioxidantes e sua biodisponibilidade na pele.

Assim, com o avanço da tecnologia, metodologias cada vez mais modernas estão disponíveis para avaliação de antioxidantes cujo uso é consagrado, ou ainda para estudo de novas substâncias que podem ser promissoras, sendo que a própria tecnologia se torna o limite para a avaliação quantitativa dos seus efeitos reais no tratamento ou prevenção de patologias.

\section{AGRADECIMENTOS}

Às agências de fomento: FAPESP (Fundação de Amparo à Pesquisa do Estado de São Paulo), CAPES (Coordenação de Aperfeiçoamento de Pessoal de Nível Superior) e CNPq - "Instituto do Milênio - Redoxoma" (Conselho Nacional de Pesquisa e Desenvolvimento). Ao Dr. J. L. Gesztesi e à Dra. S. Hurtado, ambos da empresa Natura, pelos comentários e discussões.

\section{REFERÊNCIAS}

1. Junqueira, L. C. U.; Carneiro, J.; Histologia Básica, $5^{\mathrm{a}}$ ed., Guanabara: Rio de Janeiro, 1999.

2. Rasilainen, S.; Nieminen, J. M.; Levonen, A. L.; Otonkoski, T.; Lapatto, R.; Biochem. Pharmacol. 2002, 63, 1297.

3. Beak, S. M.; Paek, S. H.; Jahng, Y.; Lee, Y. S.; Kim, J. A.; Eur. J. Pharmacol. 2004, 498, 19. 
4. King, B. A.; Oh, D. H.; Photochem. Photobiol. 2004, 80, 1.

5. Wei, Y. H.; Pang, C. Y.; Lee, H. C.; Lu, C. Y.; Curr. Sci. 1998, 74, 887.

6. Halliwell, B.; Gutteridge, J. M. C.; Free Radicals in biology and medicine, $3^{\text {rd }}$ ed., Oxford Science Publications: Oxford, 1998.

7. Nelson, K. K.; Melendez, J. A.; Free Radical Biol. Med. 2004, 37, 768.

8. Zangar, R. C.; Davydov, D. R.; Verma, S.; Toxicol. Appl. Pharmacol. 2004, 199, 316.

9. Thorn, T. R.; Gniadecki, A. B.; Free Radical Res. 2001, 35, 405.

10. Pelle, E.; Huang, X.; Mammone, T.; Marenus, K.; Maes, D.; Frenkel, K.; J. Invest. Dermatol. 2003, 121, 177.

11. Podda, M.; Weber, C.; Traber, M. G.; Packer, L.; J. Lipid Res. 1996, 37, 893.

12. Di Mascio, P.; Medeiros, M. H. G.; Bechara, E. J. H.; Catalani, L. H.; Ciência e Cultura: J. Braz. Assoc. Adv. Sci. 1995, 47, 297.

13. Miamoto, S.; Martinez, G. R.; Medeiros, M. H. G.; Di Mascio, P.; J. Am. Chem. Soc. 2003, 125, 6172 .

14. Ravanat, J. L.; Martinez, G. R.; Medeiros, M. H. G.; Di Mascio, P.; Arch. Biochem. Biophys. 2004, 423, 23.

15. Schieke, S. M.; von Montfort, C.; Buchczyk, D. P.; Timmer, A.; GretherBeck, S.; Krutmann, J.; Holbrook, N. J.; Klotz, L. O.; Free Radical Res. 2004, 38, 729 .

16. Kohen, R.; Fanberstein, D.; Tirosh, O.; Arch. Gerontol. Geriatr. 1997, 24, 103.

17. Fridovish, I.; J. Exp. Biol. 1998, 201, 1203.

18. Rover Jr., L.; Hoehr, N. F.; Vellasco, A. P.; Kubota, L. T.; Quim. Nova 2001, 24, 112.

19. Bialy, T. L.; Rothe, M. J.; Grant-Kels, J. M.; Dermatol. Surg. 2002, 28, 1143.

20. Gandra, P. G.; Alves, A. A.; Macedo, D. V.; Kubota, L.T.; Quim Nova 2004, 27, 980 .

21. Kvam, E.; Dahle, J.; J. Invest. Dermatol. 2003, 121, 564

22. Reiter, R. J.; Adv. Pharmacol. 1997, 38, 103.

23. Slominski, A.; Pisarchik, A.; Semak, I.; Sweatman, T.; Wortsman, J.; Szczesniewski, A.; Slugocki, G.; McNulty, J.; Kauser, S.; Tobin, D. J.; Jing, C.; Johansson, O.; FASEB J. 2002, 16, 896.

24. Podda, M.; Zollner, T. M.; Grundmann-Kollmann, M.; Thiele, J. J.; Packer, L.; Kaufmann, R.; Curr. Problems Dermatol. 2001, 29, 43.

25. Sergeev, P. V.; Ukhina, T. V.; Shimanovskii, N. L.; Bull. Exp. Biol. Med. 1999, 128, 1235

26. Ichihashi, M.; Ueda, M.; Budiyanto, A.; Bito, T.; Oka, M.; Fukunaga, M.; Tsuru, K.; Horikawa, T.; Toxicology 2003, 189, 21.

27. Krokan, H. E.; Standal, R.; Slupphaug, G.; Biochem. J. 1997, 325, 1.

28. Krutmann, J. Em Photoimmunology; Krutmann, J.; Elmets, C. A., eds.; Blackwell Science: Cambridge, 1995, p. 246.

29. Lyons, N. M.; O’Brien, N. M.; J. Dermatol. Sci. 2004, 30, 73.

30. Scharffetter-Kochanek, K.; Brenneisen, P.; Wenk, J.; Herrmann, G.; Ma, W.; Kuhr, L.; Meewes, C.; Wlaschek, M.; Exp. Gerontol. 2000, 35, 307.

31. Jurkiewicz, B. A.; Buettner, G. R.; Photochem. Photobiol. 1996, 64, 918.

32. Masaki, H.; Atsumi, T.; Sakurai, H.; Biochem. Biophys. Res. Commun. 1995, 206, 474

33. Thiele, J. J.; Traber, M. G.; Packer, L.; J. Invest. Dermatol. 1998, 110, 756.

34. Yamamoto, J.; Dermatol. Sci. 2001, 27, S1.

35. Yasui, H.; Sakurai, H.; Biochem. Biophys. Res. Commun. 2000, 269, 131.

36. Clydesdale, G. J.; Dandie, G. W.; Muller, H. K.; Immunol. Cell Biol. 2001, $79,547$.

37. Wilgus, T. A.; Parrett, M. L.; Ross, M. S.; Tober, K. L.; Robertson, F. M.; Oberyszyn, T. M.; Adv. Exp. Med. Biol. 2002, 507, 85.

38. Hruza, L. L.; Pentland, A. D.; J. Invest. Dermatol. 1993, 100, 535.

39. Krutmann, J.; Grewe, M.; J. Invest. Dermatol. 1995, 105, 67s.

40. Heck, D. E.; Vetrano, A. M.; Mariano, T. M.; Laskin, J. D.; J. Biol. Chem. 2003, 278, 22432.

41. Khun, A.; Fihsel, K.; Lehmann, J.; Kuzicka, T.; Kolb-Bachofen, V.; J. Invest. Dermatol. 1998, 111, 149.

42. Nabeyrat, E.; Jones, G. E.; Fenwick, P. S.; Barnes, P. J.; Donnelly, L. E.; Am. J. Physiol. Lung Cell Mol. Physiol. 2003, 284, L1112.

43. Szabo, C.; Toxicol. Lett. 2003, 11, 105.

44. Haralampus-Grynaviski, N.; Ransom, C.; Ye, T.; Rozanowska, M.; Wrona, M.; Sarna, T.; Simon, J. D.; J. Am. Chem. Soc. 2002, 124, 3461.

45. Menon, E. L.; Morrison, H.; Photochem. Photobiol. 2002, 75, 656.

46. Stadtman, E. R.; Science 1990, 257, 1220.

47. Linton, S.; Davies, M. J.; Dean, R. T.; Exp. Gerontol. 2001, 36, 1503.

48. Draper, H.; Hadley, M.; Xenobiotica 1990, 20, 901.

49. Chaudhary, A. K.; Nokubo, M.; Reddy, G. R.; Yeola, S. N.; Morrow, J. D.; Blair, I. A.; Marnett, L. J.; Science 1994, 265, 1580.

50. Medeiros, M. H. G.; Carvalho, V. M.; Farias, L. P.; Loureiro, A. P. M.; Ciência e Cultura: J. Braz. Assoc. Adv. Sci. 1995, 47, 336.

51. Goodman, M. F.; Annu. Rev. Biochem. 2002, 71, 17.

52. Heck, D. E.; Gerecke, D. R.; Vetrano, A. M.; Laskin, J. D.; Toxicol. Appl. Pharmacol. 2004, 195, 288.
53. Einspahr, J. G.; Alberts, D. S.; Warneke, J. A.; Bozzo, P.; Basye, J.; Grogan, T. M.; Nelson, M. A.; Bowden, G. T.; Neoplasia 1999, 1, 468.

54. Renzing, J.; Hansen, S.; lane, D. P.; J. Cell Sci. 1996, 109, 1105.

55. Smith, M. L.; Chen, I. T.; Zhan, Q. M.; Oconnor, P. M.; Fornace, A. J.; Oncogene 1995, 10, 1053.

56. Brash, D. E.; Ziegler, A.; Jonason, A. S.; Simon, J. A.; Kunala, S.; Leffell, D. J.; J. Invest. Dermatol. Symp. Proc. 1996, 1, 136.

57. Chang, H.; Sander, C. S.; Muller, C. S.; Elsner, P.; Thiele, J. J.; Biol. Chem. 2002, 383, 703.

58. Giannetti, L.; Consolo, U.; Magnoni, C.; Muzio, L. L.; Oncol. Rep. 2004 $11,401$.

59. Lawley, W.; Doherty, A.; Denniss, S.; Chauhan, D.; Pruijn, G.; van Venrooij, W. J.; Lunec, J.; Herbert, K.; Rheumatology 2000, 39, 253.

60. Núñez-Sellés A. J.; J. Braz. Chem. Soc. 2005, 16, 699.

61. Burton, G. W.; Traber, M. G.; Acuff, R. V.; Walters, D. N.; Kay-den, H.; Hughes, L.; Ingold, K.; Am. J. Clin. Nutr. 1998, 67, 669.

62. Thiele, J. J.; Weber, S. U.; Packer, L.; J. Invest. Dermatol. 1999, 133, 1006.

63. Nachbar, F.; Korting, H. C.; J. Mol. Med. 1995, 73, 7.

64. Passi, S.; De Pita, O.; Puddu, P.; Littarru, G. P.; Free Radical Res. 2002, 36,417

65. Roxborough, H. E.; Burton, G. W.; Kelly, F. J.; Free Radical Res. 2000 , $33,437$.

66. Vaule, H.; Leonard, S. W.; Traber, M. G.; Free Radical Biol. Med. 2004 , 36,456

67. McVean, M.; Liebler, D. C.; Carcinogenesis 1997, 18, 1617.

68. McVean, M.; Liebler, D. C.; Mol. Carcinog. 1999, 24, 169.

69. van Haaften, R. I. M.; Evelo, C. T. A.; Penders, J.; Eijnwachter, M. P. F.; Haenen, G. R. M. M.; Bast, A.; Biochem. Biophys. Acta 2001, 1548, 23.

70. Mitchel, R. E.; McCann, R.; Carcinogenesis 1993, 14, 659.

71. Thornton, D. E.; Jones, K. H.; Jiang, Z. C.; Zhang, H. F.; Liu, G. F.; Cornwell, D. G.; Free Radical Biol. Med. 1995, 18, 963.

72. F'guyer, S.; Afaq, F.; Mukhtar, H.; Photodermatol. Photoimmunol. Photomed. 2003, 19, 56.

73. Fuchs, J.; Kern, H.; Free Radical Biol. Med. 1998, 25, 1006.

74. Keller, K. L.; Fenske, N. A.; J. Am. Acad. Dermatol. 1998, 39, 611.

75. Mireles-Rocha, G. H.; Galindo, I.; Huerta, M.; Trujillo-Hernandez, B.; Elizalde, A.; Cortes-Franco, R.; Acta Derm. Venereol. 2002, 82, 21.

76. Murray, J.; Darr, D.; Reich, J.; J. Invest. Dermatol. 1991, 96, 587.

77. Murray, J.; Darr, D.; Reich, J.; Pinnell, S. R.; Clin. Res. 1992, 40, 143A.

78. Bissett, D. L.; Chatterjee, R.; Hannonen, D. P.; Photodermatol. Photoimmunol. Photomed. 1990, 7, 56.

79. Darr, D.; Combs, S.; Dunston, S.; Manning, T.; Pinell, S.; Br. J. Dermatol. 1992, 127, 247.

80. Darr, D. J.; Colven, R. M.; Pinnell, S. R. Em Vitamin C in health and disease; Packer, L.; Fuchs, J., eds.; Marcel Dekker: New York, 1997, p. 517.

81. Minoti, G.; Aust, S. D.; Lipids 1992, 27, 219.

82. Lanigan, R. S.; Int. J. Toxicol. 1999, 18, 1.

83. Wrona, M.; Rozanowska, M.; Sarna, T.; Free Radical Biol. Med. 2004, 36 , 1094.

84. Edge, R.; McGarvey, D. J.; Truscott, T. G.; J. Photochem. Photobiol., B 1997, 41, 189.

85. Packer, J. E.; Slater, T. F.; Willson, R. L.; Nature 1979, 278, 737.

86. Krinsky, N.; Ann. N. Y. Acad. Sci. 1998, 20, 443.

87. Liebler, D.; McClure, T.; Chem. Res. Toxicol. 1996, 9, 8

88. Montenegro, M.; Nazareno, M.; Durantini, E.; Borsarelli, C.; Photochem. Photobiol. 2002, 75, 353.

89. Cardoso, S. L.; Quim Nova 1997, 20, 535

90. Di Mascio, P.; Kaiser, S.; Sies, H.; Arch. Biochem. Biophys. 1989, $274,532$.

91. Tapiero, H.; Townsend, D. M.; Tew, K. D.; Biomed. Pharmacother. 2004, 58,100

92. Lowe, G. M.; Booth, L. A.;Young, A. J.; Bilton, R. F.; Free Radical Res. 1999, 30, 141.

93. Palozza, P.; Nutr. Rev. 1998, 56, 257.

94. Young, A. J.; Lowe, G. M.; Arch. Biochem. Biophys. 2001, 385, 20.

95. Wang, X. D.; Russel, R. M.; Nutr. Rev. 1999, 57, 263.

96. Andreassi, M.; Stanghellini, E.; Ettorre, A.; Di Stefano, A.; Andreassi, L.; Eur. Acad. Dermatol. Venereol. 2004, 18, 52.

97. Cordova, C. A. S.; Siqueira, I. R.; Netto, C. A.; Yunes, R. A.; Volpato, A. M.; Filho, V. C.; Curi-Pedrosa, R.; Creczynski-Pasa, T. B.; Redox Rep. 2002, 7, 95

98. Gomes, A. J.; Lunardi, C. N.; Gonzalez, S.; Tedesco, A. C.; Braz. J. Med. Biol. Res. 2001, 34, 1487.

99. Ropke, C. D.; Meirelles, R. R.; Silva, V. V.; Sawada, T. C. H.; Barros, S. B. M.; Photochem. Photobiol. 2003, 78, 436.

100. Alexis, A. F.; Jones, V. A.; Stiller, M. J.; Int. J. Dermatol. 1999, 38, 735

101. Katikyar, S. K.; Yoshida, Y.; Matsui, M. S.; Cooper, K. D.; Mukhtar, H.; Photochem. Photobiol. 1999, 69S. 
102. Katiyar, S. K.; Matsui, M. S.; Elmets, C. A.; Mukhtar, H.; Photochem. Photobiol. 1999, 69, 148.

103. Kim, S. J.; Lim, M. H.; Won, Y. H.; Skin Pharmacol. 1997, 10, 200.

104. Kim, S. J.; J. Dermatol. 2001, 28, 193.

105. Parsad, D.; Pandhi, R.; Juneja, A.; Clin. Exp. Dermatol. 2003, $28,285$.

106. Afaq, F.; Adhami, V. M.; Ahmad, N.; Mukhtar, H.; Front. Biosci. 2002, 7 , d784.

107. Steenvoorden, D. P. T.; van Henegouwen, G. M. J. B.; J. Photochem. Photobiol. 1997, 41, 1 .

108. Vertuani, S.; Ziosi, P.; Salaroli, N.; Buzzoni, V.; Carli, M.; Lucchi, E.; Valgimigli, L.; Baratto, G.; Manfredini, S.; Skin Res. Technol. 2003, 9, 245.

109. Simonsen, L.; Jørgensen, A.; Benfeldt, E.; Groth, L.; Eur. J. Pharm. Sci. 2004, 21, 379.

110. Kohen, R.; Gati, I.; Toxicology 2000, 148, 149.

111. Lomuto, M.; Pellicano, R.; Giuliani, M.; Clin. Dermatol. 1995, 13, 409.

112. Alho, H.; Leinonen, J.; Methods Enzymol. 1999, 299, 3.

113. Ou-Yang, H.; Stamatas, G.; Saliou, C.; Kollias, N.; J. Invest. Dermatol. 2004, 122, 1020.

114. Evelson, P.; Ordonez, C. P.; Llesuy, S.; Boveris, A.; J. Photochem. Photobiol., B 1997, 38, 215.

115. Sauermann, G.; Mei, W. P.; Hoppe, U.; Stab, F.; Methods Enzymol. 1999, $300,419$.
116. Ermakov, I. V.; Ermakova, M. R.; Gellermann, W.; Lademann, J.; J. Biomed. Optics 2004, 9, 332

117. Kreilgaard, M.; Adv. Drug Del. Rev. 2002, 54, S99.

118. Leveque, N.; Robin, S.; Muret, P.; Mac-Mary, S.; Makki, S.; Berthelot, A.; Kantelip, J. P.; Humbert. P.; Dermis Acta Derm. Venereol. 2004, 84, 2.

119. Guaratini, T.; Vessecchi, R. L.; Lavarda, F. C.; Campos, P. M. B. G.; Naal, Z.; Gates, P. J.; Lopes, N. P.; Analyst 2004, 129, 1223.

120. Gundersen, T. E.; Blomhoff, R.; J. Chromatogr., A 2001, 935, 13.

121. van Berkel, G. J.; Zhou, F.; Anal.Chem. 1994, 66, 3408.

122. van Berkel, G. J.; McLuckey, A. S.; Glish, G. L.; Anal. Chem. 1992, 64, 1586.

123. Jurkiewicz, B. A.; Buettner, G. R.; Photochem. Photobiol. 1994, 59, 1.

124. Nishi, J.; Ogura, R.; Sugiyama, M.; Hidaka, T.; Kohno, M.; J. Invest. Dermatol. 1991, 97, 115

125. Ogura, R.; Sugiyama, M.; Nishi, J.; Haramaki, N.; J. Invest. Dermatol. 1991, 97, 1044

126. Herrling, T.; Fuchs, J.; Rehberg, J.; Groth, N.; Free Radical Biol. Med. $\mathbf{2 0 0 3}, 35,59$.

127. http://www.appliedbiosystems.com/europe/biosystems/pdf/iss $11 /$ biosol_iss11_omaldi_qstar.pdf, acessada em Janeiro 2005. 